\title{
Experience of Protagonists in Workplace Bullying: An Integrated Literature Review
}

\section{April L. Jones}

School of Psychology, Walden University,100 S Washington Ave \#900, Minneapolis, MN 55401, USA

\begin{abstract}
Awareness of workplace bullying is increasing due to an improved understanding of the psychological, social, and organizational consequences of these behaviors. In the field of nursing, which has been facing critical shortages for decades, workplace bullying has been blamed for higher attrition rates, reduced productivity, and even the current nursing shortage. Poorer patient care and reduced safety on the job are also noted sequelae of bullying among nurses. While incidents of bullying may be an accepted though unintended consequence of power dynamics within an organizational structure, there is a critical need for managers to analyze the extent of the problem, determine its impact, and try to understand how it affects staff satisfaction and retention. Although there is evidence that lack of managerial support and hierarchical work environments that disempower staff contribute to bullying among nurses, there does not appear to be agreement on the most important factors that contribute to the perpetuation of these harmful behaviors, or more importantly, on how to reduce the frequency and impact of bullying events. A number of studies have been conducted on workplace bullying; however, very few in the field of nursing have been undertaken. This paper systematically examines the impact of bullying, the factors that perpetuate its continuance, and synthesizes the literature on promising environmental and individual-level interventions in the field of nursing.
\end{abstract}

\section{Introduction}

Research has shown that bullying is a serious issue among those in the nursing profession. Estimates of nurses' exposure to abuse, bullying, violence and harassment range from $27 \%$ - $80 \%[1,2]$. Perpetrators may be patients, nurse colleagues, physicians or others, but the evidence suggests that the main perpetrators are nurses in senior positions and those who are established staff members $[1,3]$. The focus of this research paper is on the impact of bullying, the factors that contribute to its continuance and the interventions that may be initiated to help mitigate the problem.

According to the psychologist, Vartia [5], the behavior of individuals changes little as they grow older, suggesting that being a bully during childhood is likely strongly predictive of being a bully as an adult. Workplace bullying represents a chain reaction, usually initiated by incivility, followed by bullying, and culminates in violence at the place of work [6]. Some examples of the types of bullying behaviors seen within organizations include serial bullying, perpetrated by individuals who often lie and manipulate to avoid consequences, and gang bullying, perpetrated by a group, and often called "mobbing." Although many companies have policies that help prevent workplace bullying, a large number do not enforce them [7].

Despite the frequent occurrence of the phenomenon, no comprehensive national legislation has been enacted in the U.S. to help mitigate and eradicate its occurrence. In contrast, other developed countries, such as the U.K., have national policies to protect and defend victims and witnesses. However, since 2002, a group of grassroots activists have been working to ensure passage of the Healthy Workplace bill in each state (The Healthy Workplace Campaign, n.d.). To date, 30 state legislatures and two territories have introduced the bill which creates a platform for victims of bullying to pursue a legal solution if they have proof of malicious actions targeted towards them.

For bullying to begin and remain a significant force within an organization, a number of factors must be in place to allow for its perpetuation. For instance, bystanders who are either apathetic or fearful of repercussion, or worse, collude with the instigators, enabling structures within an organization, and the lack of efficient communication channels that allow employees to be harassed without intervention, all lead to a situation where bullying thrives [8-10]. These conditions allow for the cultivation of an environment of incivility that fuels the bullying behavior and victimizes the targets [9].

\section{Methodology}

According to Torraco [11], an integrative literature review is designed to utilize existing information on a given topic with the aim of generating a new perspective or framework. To this extent therefore, this paper focuses on the analysis and synthesis of existing data within the field of workplace bullying. This paper draws on a comprehensive review of peer-reviewed articles, both international and domestic, that covers bullying in the nursing profession.

Peer-reviewed published articles in the area of nurse bullying published after 2010 were included in this review. Studies were included if they covered any aspect of this phenomenon, focusing on the impact of bullying, the environmental and human factors that allow for its continuance, and interventions to reduce impact. Special attention was placed on articles that attempted to examine bullying as a social phenomenon, therefore case studies or studies with very few participants were excluded.

"Corresponding Author: Dr. April L. Jones, School of Psychology, Walden University,100 S Washington Ave \#900, Minneapolis, MN 55401, USA, E-mail: april.jones2@waldenu.edu

Citation: Jones AL (2017) Experience of Protagonists in Workplace Bullying: An Integrated Literature Review. Int J Nurs Clin Pract 4: 246. doi: https://doi. org/10.15344/2394-4978/2017/246

Copyright: () 2017 Banakhar et al. This is an open-access article distributed under the terms of the Creative Commons Attribution License, which permits unrestricted use, distribution, and reproduction in any medium, provided the original author and source are credited. 
The literature search strategy included comprehensive searches in PubMed, Google Scholar, and PsycInfo. The search terms included nurses and bullying, bully, or bullies. The initial review included a determination, based on the title of the articles, of whether the article was relevant. After this initial selection, abstracts were reviewed to determine if the article included an examination of the phenomenon, as noted above. After narrowing down the abstracts based on these criteria, full articles were reviewed.

For the purpose of coherence within this paper, a single and unified definition of the phenomenon was utilized. Workplace bullying involves the continuous perpetration of negative acts on a victim accruing from an imbalance of power between the perpetrator and victim, often resulting in physical and psychological harm [12].

\section{Results}

\section{Impact of bullying}

The literature has demonstrated that nursing staff are more likely to experience uncivil behaviors and bullying compared to other healthcare professionals [13]. Bullying has an impact at both the organizational and individual levels, though research in the field has focused primarily on the individual-level impacts. Exposure to bullying and other uncivil behaviors is a predictor of nurses' intention to stay on the job [13]. Bullying has been found to be related to job satisfaction, burnout, commitment to work, and intention to leave $[1,14-18$,]. One study estimated that up to $25 \%$ of nurses who are bullied actually leave the profession [3].

At the organizational level, poorly organized places of work increase the prevalence of negative behaviors, leading to increased turnover rates, lower workplace morale, and decreased revenues [19]. Psychologically-related medical conditions may become more common, thereby increasing the company's costs of production $[20,21$,$] . In the event that employees choose to legally address their$ plight, worker compensation payouts will also increase company costs [22].

On the individual level, the impact of bullying on a target may be psychological or physiological and almost certainly will reduce job performance $[23,24]$. Bullies' communications demean their victims, shame them, and make them less likely to speak up [25,]. However, bullying tends to affect even the non-targeted employees of an organization [26]. Olender-Russo [27] asserts that employees who witness incidents of negative behavior at their places of work tend to suffer similar sequelae compared to the actual targets of bullying.

Bullying has been associated with a greater number of health complaints and more mental health symptoms, including perceived stress, anxiety, and symptoms of post-traumatic stress disorder, all of which may reduce quality of life and negatively affect patient care and safety $[2,14,16,28,29]$. Poor sleep, distress, depression, poor work attitudes, and ineffective communication with other staff members are often the outcomes of bullying $[3,17,30]$. It has also been suggested that bullying may not affect health directly, but rather indirectly, mediated by burnout [31].

These impacts have a significant effect on victims of bullying, however there are other victims who themselves may not have been the direct target of bullies. These include witnesses who may experience some of the same discomfort as the actual victims, and who may also experience fear of retaliation. The evidence suggests this fear may help to explain why some witnesses fail to help the victims of bullying, even when they want to help [32].

The evidence suggests that the support of family and friends may serve to reduce the impact of bullying and that being a parent may serve as a protective factor for victims [33]. A study in India among nursing students suggested that most students did not have the means to cope with bullying behavior [34]. On the other hand, a U.K. study found that some nursing students followed up the incidents of bullying with questions and a challenge, unlike the majority of the medical students who generally accepted the bullying without defending themselves [35]. These data indicate that bullying behaviors appear to be common enough in the nursing profession to warrant serious focus from managerial and supervisory staff.

Senior nurses and those in supervisory positions are often the perpetrators of bullying, though there is a vast range of individuals who engage in bullying behaviors. A study in Singapore found that some senior nurses used the euphemism "tough love," though the authors more appropriately termed the behavior "abusive," to rationalize their negative behaviors towards new nurses [36]. Though these behaviors negatively affect the transition of new nurses into practice and may influence their willingness to remain with the facility and even in the profession, these behaviors continue to be perpetrated.

\section{Factors that allow continuance}

There are a number of factors-interpersonal and environmental that contribute to the continuation of bullying behaviors in nursing, though there is strong evidence that the work environment, rather than individual characteristics may have a greater impact on bullying and its prevention [37]. On the environmental side, organizational culture and the characteristics of the job affect the extent to which bullying is allowed to thrive in the workplace [38]. The factor most discussed in the literature as a contributory factor to bullying is the existence of a hierarchical, as opposed to a relation-oriented, work environment $[3,39,40$,$] . Bullying often has been reported as more$ likely to be vertical, from higher level to lower level staff $[4,15]$.

A bullying culture, that is, one where the behavior is known to exist and appears to be accepted by those in power, disempowering work environments, a lack of teamwork, and the sense that the workplace is not safe contribute to bullying $[3,15,39,41]$. In addition, when job characteristics are not well-defined and when organizational processes are misused, bullying occurs $[42,43]$. Bullying also seems to increase with lack of sufficient staff resources or a nursing shortage $[15,44]$.These factors suggest strongly that there is a need to revise the organizational processes that contribute to bullying [43].

Interpersonal contributors to bullying include the presence of informal negative alliances that serve to isolate and intimidate those who are not members of the clique [43]. Leadership styles that may be more hierarchical and not perceived as supportive may encourage bullying $[4,15,38]$. In addition, the quality of staff relationships affect the extent to which bullying is allowed to thrive in the workplace [38].

There appears to be a lack of training and standardization for supervisors on how to manage bullying behaviors on the job, although supervisors who have witnessed, been victims or supervised nurses for more than 20 years were more likely to recognize and act upon 
bullying behaviors [45]. It was interesting to note that being able to identify bullying behaviors was not strongly correlated with responding to these behaviors [45]. When managers are silent, do nothing, when supervision is abusive, or when there are no support structures for victims, these behaviors flourish $[3,42,46]$. In many cases, policies are not clear on how managers should respond to bullying, suggesting that managers may not have sufficient guidance, and may enforce policies without standardization [47].

\section{Interventions to address bullying}

There are no standard, or universally accepted approaches to reduce the incidence and impact of bullying among nurses. A review of the literature over the past 30 years shows that very few interventions have been developed to address the problem of bullying or the power dynamics that cause these behaviors to begin. It is clear that while some nurse managers have the experience and/or training to address workplace bullying, others may not be equipped to resolve the issues [48].

It is clear that bringing the issues out into the open, allowing for discussion and staff input, and incorporating conflict management may reduce the problems $[49,50]$. Trying to strengthen the relationship between leaders and staff, utilizing transformational and authentic leadership styles, and empowering staff to improve the work environment has also been associated with reduced bullying behaviors [50,51]. On the interpersonal side, there must be an effort to resolve interpersonal conflicts that might escalate to bullying, and individuals must be held accountable for their behaviors [16,41].

In terms of individual level interventions, there have been attempts to use cognitive rehearsal, which allows an individual to practice how they would react in specific situations, as well as incorporating assertiveness and aggression training for the victims [52,53].This type of intervention has been successful in reducing incivility in the workplace.

A critical factor in the continuance of bullying is the fear of retaliation among witnesses who want to, but who are afraid of reporting these negative behaviors. Therefore, efforts must be made to reduce the fear of retaliation if witnesses intervene. Without this approach, even though many witnesses want to help victims, their intention to help is often not converted to concrete behaviors because of fear of retaliation [54].

A critical factor in the continuance of bullying is the fear of retaliation among witnesses who want to, but who are afraid of reporting these negative behaviors. Therefore, efforts must be made to reduce the fear of retaliation if witnesses intervene. Without this approach, even though many witnesses want to help victims, their intention to help is often not converted to concrete behaviors because of fear of retaliation [55].

Making it clear to all staff what types of behaviors are unacceptable, though a seemingly simple and obvious step, is often not undertaken in many workplaces. A program in the U.S. developed a comprehensive code of conduct regarding uncivil behaviors. A study conducted before and after implementation of the code resulted in the perception of significant reduction in uncivil behaviors as a result of establishment of the codes of conduct [56]. In addition, a zero-tolerance policy, clear and prompt responses by managers, and policies, performance reviews and education have all been shown to reduce the incidence of bullying [3,57].
Consistent and continuous efforts to measure and respond to the perception of bullying behaviors may reduce the incidence of bullying. For example, the Negative Acts Questionnaire-Revised (NAQ-R), a widely-used instrument, has been utilized by organizations attempting to assess and develop interventions to address bullying [13]. Without an accurate understanding of the incidence and impact of bullying, management may not be aware of the extent of the problem, and will have no metrics to determine if any improvements have occurred.

\section{Recommendations}

Although the phenomena of bullying in nursing are not new, very few interventions have been systematically studied and published in the literature. Due to this shortcoming, much of the intervention literature is "grey" literature, which has been published by agencies and organizations, with the U.K. in the lead of national approaches to the issue. For example, the Royal College of Nursing [58] in the U.K. published best practice guidelines that include having a written policy on bullying and harassment in the workplace, and ensuring that these policies are communicated to employees when they first join the organization and at regular intervals thereafter. The College also strongly advises against ignoring complaints, delay, or preventing or discouraging staff from reporting incidents. Many of these same recommendations are repeated in a Northern Ireland publication [59].

The National Health Service (NHS) of Scotland [60] offers a "Dignity at Work" toolkit that focuses on five main concepts, PartnershipUnderstanding, Relations, Environment, and Leadership-to promote respect among all workers and to tackle the issue of bullying. The toolkit not only focuses on reducing negative aspects of the environment, but also on building a strong foundation, thereby enhancing employees' sense of pride and motivation, and potentially reducing poor work performance and turnover. This toolkit, along with the IMatter, which is a tool designed for continuous improvement and was developed in cooperation with staff at the NHS in Scotland (n.d) [61], to improve staff experience, as well as recently developed comprehensive guidance for employers [62] may serve as models for interventions in the U.S.

Efforts to address bullying should start at the very beginning of a new nurse's career, when bullying often arises. A recommended intervention would be a formal program that reduces the impact of bullying as new nurses transition to their new roles and improves the experience of moving from student to practitioner [63].

A viable solution to workplace bullying may lie in the development of anti-bullying apps. The app should focus on ways to mitigate bullying. The app should incorporate a feature of anonymous alerts to the human resources department. This approach was used successfully by Newtown Public Schools [64] (Newtown Public School District Chooses Anonymous Alerts Safety and Bullying Reporting App, n.d). The school opted to use the Anonymous Alerts app (http:// www.anonymousalerts.com/webcorp/) to deal with bullying behavior within the institution while maintaining the confidentiality of the reporting party.

A number of apps have been developed to reduce the impact of bullying, but most of these have been developed for schools and for children, for example, Anonymous Alerts ${ }^{\oplus}$ and Speak UP! $!^{\oplus}$ (https:// www.gospeakup.com/pri_sec/). In the workplace, one app, STOPit PRO (https://stopitcyberbully.com/workplace/), empowers each employee to monitor compliance with rules and utilizes anonymous 
reporting to managers. It is not specifically designed for bullying, but is targeted to all types of inappropriate behaviors. It is marketed not only to deter unethical behaviors but also to mitigate risk to the companies that use it.

The CRANAplus Bullying app (https://crana.org.au/support/ bullying-app) helps customers to identify the symptoms of bullying and facilitates the creation of an action plan to deal with the situation. It also offers a direct way to access support. It is free to remote and rural health practitioners. The program offers free phone counseling and support to health providers and families working in rural areas and also offer workshops on managing stress, counseling and other services. Bully Proof Assistant (https://play.google.com/store/apps/ details?id=com.robertrichardson.bullyproofassistantLite\&hl=en), documents incidents of workplace bullying, enabling users to use the information for legal or employment cases. Users may also use the app to send incident reports. These and other similar apps may be the next wave in helping to reduce the incidence of bullying in the workplace.

Based on this review of the literature, a number of recommendations are made. It is critically important for the workplace to have policies that are communicated early on and continuously updated and reviewed. Supervisors must be trained to recognize the signs of bullying and harassment behaviors and taught how to intervene. Trainings must be very specific, for example, when a bully sabotages work, or yells, or demeans a co-worker, what actions should be taken? What types of interviewing techniques should be utilized to help ensure honesty from a bully? What kinds of corroborating evidence should be required, particularly if the bully has been careful enough to ensure that there were no witnesses? The nursing profession might take some guidance from psychology, with the understanding that bullying is generally an ingrained behaviour pattern that the perpetrator has perfected over the years. The bully is likely to offer a number of rationalizations for his/her behaviour, and thus it is critically important for human resource departments and supervisors to know the tell-tale signs to look for-in the bully, as well as in victims and witnesses. Therefore, training should be in-depth; a simple oneoff training will not be sufficient to address the widespread and insidious nature of this problem.

Employees and witnesses must feel empowered to report incidents of bullying, as the evidence suggests that many witnesses do not speak up due to fear of repercussion. This might mean making it easier to report bullying, perhaps anonymously, by use of an app or distress button in the office, or a code word to security or another designated person in the building. Most importantly, individuals must have trust in the system. If there is a designated person or supervisor to whom bullying will be reported, they must be perceived as trustworthy and willing to challenge ingrained organizational dysfunction, to ensure that workers are heard and are treated fairly.

\section{Conclusions}

The findings of this integrative review support the existence of bullying as a significant issue in nursing that leads to low morale, high stress and the potential for high turnover among nursing staff [14], however there is little agreement about how best to approach and resolve the problem. The evidence suggests that organizational, rather than individual-level interventions may have the greatest chance for success, since the factors that allow for this behavior to begin and to persist are facilitated by organizational culture, lack of awareness of the extent of the problem, and gaps in experience or training on how to deal with the issue of bullying.
This review reveals that while there are quite a number of articles on the subject, there are very few systematic studies of interventions and the extent to which they are successful. As a result of this gap in the peer-reviewed literature, this review includes a number of interventions and recommendations from grey literature and organizations. Though there are interventions currently being used in practice domestically and worldwide, the lack of rigorous outcomes testing presents a significant gap as well as an opportunity for researchers.

Interventions should be preceded by an assessment to determine the scope of the problem. Facing hard truths about those who are the bullies, who may be persons of power, will necessitate the development of codes of conduct, training, and constant review to ensure that policies are enforced. The front line in the interventions will be supervisory staff paying attention to their leadership styles, for example, transformational and authentic, ensuring they have the training to recognize bullying when they see it, and setting up structures to ensure they have the support to carry out their duties may reduce the incidence of bullying. At the staff level, ensuring that staff feels empowered and that witnesses do not fear retaliation will also improve work conditions.

\section{Results}

Employing the integrative literature review approach may have been the appropriate method for this research; it, however, introduces a number of limitations. Originality, which forms the primary basis of research, is limited to the analysis and synthesis of the works of other researchers. In addition, the focus necessarily relied on international sources in order to have the most comprehensive overview of the situation. This integrative approach offers an effective way of presenting and synthesizing information to be most useful for those who may benefit from the research.

\section{Competing Interests}

The author declares that she has no competing interests.

\section{References}

1. Chang HE, Cho SH (2016) Workplace Violence and Job Outcomes of Newly Licensed Nurses. Asian Nurs Res (Korean Soc Nurs Sci) 10: $271-$ 276.

2. Sauer PA, McCoy TP (2016) Nurse Bullying : Impact on Nurses' Health. West J Nurs Res.

3. Wilson $\mathrm{JL}$ (2016) An exploration of bullying behaviours in nursing: a review of the literature. Br J Nurs 25: 303-306.

4. Norton P, Costa V, Teixeira J, Azevedo A, Roma-Torres A, et al. (2017) Prevalence and Determinants of Bullying Among Health Care Workers in Portugal. Workplace Health Saf 65: 188-196.

5. Vartia MA (2001) Consequences of workplace bullying with respect to the well-being of its targets and the observers of bullying. Scand J Work Environ Health 27: 63-69.

6. Namie G (2003) Workplace bullying: Escalated incivility. Ivey Business Journal: Improving the Practice of Management 68: 1-6.

7. Rayner $\mathrm{C}$, Hoel H, Cooper C (2001) Workplace bullying: What we know, who is to blame and what can we do?Boca Raton, FL: CRC Press.

8. Healthy Workplace Campaign. (n.d.).

9. Lewis D, Sheehan M, Davies C (2008) Uncovering workplace bullying. Journal of Workplace Rights 13: 281-301. 
Citation: Jones AL (2017) Experience of Protagonists in Workplace Bullying: An Integrated Literature Review. Int J Nurs Clin Pract 4: 246. doi: https://doi. org/10.15344/2394-4978/2017/246

Page 5 of 6

10. D'Cruz P (2015) Depersonalized bullying at work. New Delhi, India: Springer.

11. Van Heugten K (2011) Social work under pressure: How to overcome stress, fatigue and burnout in the workplace. London, UK: Jessica Kingsley Publishers.

12. Torraco R (2005) Writing integrative literature reviews. Human Resources Development Review 4: 1-12.

13. Einarsen S, Hoel H, Zapf D, Cooper CL (2003) The concept of bullying at work: The European tradition. In Einarsen S, Hoel H, Zapf D \& Cooper $\mathrm{CL}$, (Eds.), Bullying and emotional abuse in the workplace: International perspectives in research and practice (pp. 3-30). Abingdon, UK: Taylor \& Francis.

14. Evans D1 (2017) Categorizing the Magnitude and Frequency of Exposure to Uncivil Behaviors: A New Approach for More Meaningful Interventions. J Nurs Scholarsh 49: 214-222.

15. Jaradat $Y$, Nielsen MB, Kristensen P, Nijem K, Bjertness E, et al. (2016) Workplace aggression, psychological distress, and job satisfaction among Palestinian nurses: A cross-sectional study. Appl Nurs Res 32: 190-198.

16. Tong $M$, Schwendimann $R$, Zúñiga $F(2017)$ Mobbing among care workers in nursing homes: A cross-sectional secondary analysis of the Swiss Nursing Homes Human Resources Project. Int J Nurs Stud 66: 72-81.

17. Berry PA, Gillespie GL, Fisher BS, Gormley DK (2016) Recognizing, Confronting, and Eliminating Workplace Bullying. Workplace Health Saf 64: 337-341. Ovayolu O, Ovayolu N, Karadag G (2014) Workplace bullying in nursing. Workplace Health Saf 62: 370-374.

18. Ovayolu O, Ovayolu N, Karadag G (2014) Workplace bullying in nursing Workplace bullying in nursing 62: 370-374.

19. Allen BC, Holland P, Reynolds R (2015) The effect of bullying on burnout in nurses: the moderating role of psychological detachment. J Adv Nurs 71: 381-390.

20. Einarsen S, Hoel H, Notelaers G (2009) Measuring exposure to bullying and harassment at work: Validity, factor structure and psychometric properties of the Negative Acts Questionnaire-Revised. Work \& Stress 23: 24-44.

21. Gardner S, Johnson PR (2001) The leaner, meaner workplace: Strategies for handling bullies at work. Employment Relations Today 28: 23-36.

22. Johnson SL (2009) International perspectives on workplace bullying among nurses: a review. Int Nurs Rev 56: 34-40.

23. Randle J, Stevenson K, Grayling I (2007) Reducing workplace bullying in healthcare organisations. Nurs Stand 21: 49-56.

24. Zapf D, Knorz C, Kulla M (1996) On the relationship between mobbing factors, and job content, social work environment, and health outcomes. European Journal of Work \& Organizational Psychology 5: 215-237.

25. Inceoglu I (2002) Organizational culture, team climate, workplace bullying and team effectiveness: An empirical study on their relationship. Munich, Germany: Herbert Utz Verlag Wissenschaft.

26. Dzurec LC, Kennison M, Albataineh R(2014) Unacknowledged threats proffered "in a manner of speaking": Recognizing workplace bullying as shaming. J Nurs Scholarsh 46: 281-291.

27. Lewis SE, Orford J (2005) Women's experiences of workplace bullying: Changes in social relationships. Journal of Community \& Applied Social Psychology 15: 29-47.

28. Olender-Russo L1 (2009) Creating a culture of regard: an antidote for workplace bullying. Creat Nurs 15: 75-81.

29. Oh H, Uhm DC, Yoon YJ (2016) Workplace bullying, job stress, intent to leave, and nurses' perceptions of patient safety in South Korean hospitals. Nurs Res 65: 380-388.

30. Berry PA, Gillespie GL, Fisher BS, Gormley DK, Haynes JT (2016) Psychological distress and workplace bullying among registered nurses. Online J Issues Nurs 21: 8.

31. Karatas H1, Ozturk C, Bektas M (2016) A Study of Bullying Against Nursing Students. J Nurs Res

32. Giorgi G, Mancuso S, Fiz Perez F, Castiello D'Antonio A, Mucci N, et al. (2016) Bullying among nurses and its relationship with burnout and organizational climate. Int J Nurs Pract 22: 160-168.
33. Houck NM, Colbert AM (2016) Patient safety and workplace bullying: An integrative review. J Nurs Care Qual 32: 164-171.

34. Karatza C, Zyga S, Tziaferi S, Prezerakos P (2016) Workplace bullying among the nursing staff of Greek public hospitals. Workplace Health \& Safety. Ann Gen Psychiatry 15: 7.

35. Prasad K (2014) Bullying from nursing: Students' perspective. Nurs J India 105: 86-89.

36. Timm A (2014) 'It would not be tolerated in any other profession except medicine': survey reporting on undergraduates' exposure to bullying and harassment in their first placement year. BMJ Open 4: e005140.

37. Leong YM, Crossman J2 (2016) Tough love or bullying? New nurse transitional experiences. J Clin Nurs 25: 1356-1366.

38. Ganz FD, Levy H, Khalaila R, Arad D, Bennaroch K, et al. (2015) Bullying and Its Prevention Among Intensive Care Nurses. J Nurs Scholarsh 47: 505-511.

39. Trépanier SG, Fernet C, Austin S, Boudrias V (2016) Work environment antecedents of bullying: A review and integrative model applied to registered nurses. Int J Nurs Stud 55: 85-97.

40. Longo J (2013) Bullying and the older nurse. J Nurs Manag 21: 950-955.

41. An Y, Kang J (2016) Relationship between organizational culture and workplace bullying among Korean nurses. Asian Nurs Res (Korean Soc Nurs Sci) 10: 234-239.

42. Myers G, Côté-Arsenault D, Worral P, Rolland R, Deppoliti D, et al. (2016) A cross-hospital exploration of nurses' experiences with horizontal violence. $J$ Nurs Manag 24: 624-633.

43. Vagharseyyedin SA (2016) Nurses' perspectives on workplace mistreatment: A qualitative study. Nurs Health Sci 18: 70-78.

44. Blackstock S, Harlos K, Macleod, ML, Hardy CL (2015) The impact of organisational factors on horizontal bullying and turnover. J Nurs Manag 23: 1106-1114.

45. Bortoluzzi G, Caporale L, Palese A (2014) Does participative leadership reduce the onset of mobbing risk among nurse working teams? J Nurs Manag 22: 643-652.

46. Gilbert RT, Hudson JS, Strider D (2016) Addressing the elephant in the room: Nurse manager recognition of and response to nurse-to-nurse bullying. Nurs Adm Q 40: E1-E11.

47. Rodwell J, Brunetto Y, Demir D, Shacklock K, Farr-Wharton R (2014) Abusive supervision and links to nurse intentions to quit. J Nurs Scholarsh 46: 357-365.

48. Johnson SL, Boutain DM, Tsai JH, de Castro AB (2015) Managerial and Organizational Discourses of Workplace Bullying. J Nurs Adm 45: 457-461.

49. Lindy C, Schaefer $F(2010)$ Negative workplace behaviours: An ethical dilemma for nurse managers. J Nurs Manag 18: 285-292.

50. Strandmark KM, Rahm G, Wilde Larsson B, Nordstrom G, Rystedt I (2016) Preventive strategies and processes to counteract bullying in health care settings: Focus group discussions. Issues Ment Health Nurs 38: 113-121.

51. Kaiser JA(2016) The relationship between leadership style and nurse-tonurse incivility: Turning the lens inward. J Nurs Manag 25: 110-118.

52. Read E, Laschinger HK (2015) Correlates of New Graduate Nurses' Experiences of Workplace Mistreatment. J Nurs Adm 45: S28-35.

53. Griffin M, Clark CM (2014) Revisiting cognitive rehearsal as an intervention against incivility and lateral violence in nursing: 10 years later. J Contin Educ Nurs 45: 535-542.

54. Etienne E (2014) Exploring workplace bullying in nursing. Workplace Health Saf 62: 6-11.

55. Báez-León C, Moreno-Jiménez B, Aguirre-Camacho A, Olmos R (2016) Factors influencing intention to help and helping behavior in witnesses of bullying in nursing settings. Nursing Inquiry 23: 358-367.

56. Authement R (2016) Can a comprehensive code of conduct discourage incivility in nursing students? Nursing 46: 14-19.

57. Parker KM, Harrington A, Smith CM, Sellers KF, Millenbach L (2016) Creating a nurse-led culture to minimize horizontal violence in the acute care setting: A multi-interventional approach. J Nurses Prof Dev 32: 56-63. 
Citation: Jones AL (2017) Experience of Protagonists in Workplace Bullying: An Integrated Literature Review. Int J Nurs Clin Pract 4: 246. doi: https://doi. $\operatorname{org} / 10.15344 / 2394-4978 / 2017 / 246$

58. Royal College of Nursing (2017) Bullying and harassment.

59. Cassidy F, Kremer J, McAllister M, Steele R (2006) Harassment and bullying in the workplace.

60. National Health Service of Scotland (2017) Dignity at work toolkit.

61. National Health Service of Scotland. (n.d.). IMatter Toolkit.

62. Becton JB, Gilstrap JB, Forsyth M (2017) Preventing and correcting workplace harassment: Guidelines for employers. Business Horizons 60: 101-111.

63. Rush KL, Adamack M, Gordon J, Janke R (2014) New graduate nurse transition programs: Relationships with bullying and access to support. Contemporary Nurse 48: 219-228.

64. Newtown Public School District Chooses Anonymous Alerts Safety and Bullying Reporting App (n.d.).

65. Quine $L$ (2001) Workplace bullying in nurses. Journal of Health Psychology, 6: 73-84. 